\title{
Estratificación de disfunción diastólica y patrones de llenado del ventrículo izquierdo en pacientes llevados a ecocardiografía en un centro de tercer nivel según las Guías de la Sociedad Americana de Ecocardiografía y la Asociación Europea de Imágenes Cardíacas 2016
}

Echocardiographic diastolic disfunction and left ventricle filling patterns in patients studied in a terciary level center according to American Society of echocardiography/ European association of cardiovascular imaging 2016 Guidelines

Víctor Humberto Guerrero-Legro1, Gustavo Restrepo-Molina², Elsa María Vásquez Trespalacios ${ }^{3}$

\begin{abstract}
Objective: Characterize the degree of diastolic dysfunction and left ventricular filling patterns according to the 2016 ASE Guidelines applied in a population of patients taken to echocardiography in a reference center in the city of Medellín between February and June 2017. Methodology: A cross-sectional study was performed in patients undergoing echocardiography. Patients with atrial fibrillation, pacemaker implantation, AV block, mitral ring calcification, any degree of mitral stenosis, mitral insufficiency were excluded. An initial descriptive explora-
\end{abstract}

Key words: Diastolic

dysfunction,

filling patterns,

echocardiography

Fellow Anestesia Cardiotorácica, Universidad CES, Clínica Medellín, Colombia.

Cardiólogo Ecocardiografista, Jefe de Laboratorio Ecocardiografía, Clínica Medellín, Colombia. Coordinador del Posgrado de Ecocardiografía, Universidad CES Medellín, Colombia.

Epidemiologia Clínica Universidad CES Medellín, Colombia.

Fecha de recepción: 22 de enero de 2018

Fecha de aceptación: 14 de junio de 2018

ORCID

https://orcid.org/0000-0002-0057-3058

Correspondencia:

Víctor Humberto Guerrero-Legro

Email: victorguerrero1624@hotmail.com 
tory analysis was carried out by frequencies and percentages for the categorical variables and measures of central tendency (mean or median according to the normal distribution of the data) for the quantitative variables. Subsequently, it is compared with the different degrees of diastolic dysfunction with respect to age by t-Student test and with respect to categorical variables by $\chi^{2}$. Results: A total of 610 patients were recruited, of which 75 were withdrawn from the sample by the exclusion criteria, 530 patients were included of which $198(37 \%)$ were classified as grade I diastolic dysfunction, 63 (11.7\%) grade II, 29 (5.4\%) grade III, 38 (7.1\%) undetermined and 207 (39\%) without diastolic dysfunction. The age of the patients stratified in any degree of diastolic dysfunction was significantly higher in comparison with patients without diastolic dysfunction $(p<0.001)$. Hypertension was the most important associated comorbidity in patients with diastolic dysfunction grade I (in 54\%), grade II (in 50\%), while coronary disease was the most frequent concomitant in the presence of diastolic dysfunction grade III (in $48 \%$ ). The E/e' ratio greater than 14 was present in 35\% of cases with grade II diastolic dysfunction and in $72 \%$ of cases with grade III diastolic dysfunction.Conclusion: Diastolic dysfunction is a very frequent finding (323/530, $61 \%$ ). Hypertension was the most common comorbidity associated in grades I, II of diastolic dysfunction, while coronary disease with grade III. The increase in the E/e' $>14$ ratio as an indicator of increased population pressure was present in $35 \%$ of patients with grade II diastolic dysfunction and in $72 \%$ of patients with grade III dysfunction. Diastolic function was classified as undetermined in $7.1 \%$ of the total patient population. The ASE/EACVI Guidelines allowed the classification in different degrees of diastolic dysfunction in the majority of patients evaluated ( $94 \%)$. Other methods of diagnostic evaluation are necessary to adequately classify the group of patients with indeterminate diastolic function.

\section{RESUMEN}

Objetivo: Caracterizar el grado de disfunción diastólica y patrones de llenado del ventrículo izquierdo según las Guías ASE/EACVI 2016 aplicadas en una población de pacientes llevados a ecocardiografía en un centro de referencia de la ciudad de Medellín entre febrero y junio de 2017. Metodología: Se realizó un estudio de corte transversal en pacientes llevados a ecocardiografía. Fueron excluidos pacientes con fibrilación auricular, implante de marcapasos, bloqueo $A V$, calcificación del anillo mitral, cualquier grado de estenosis mitral, insuficiencia mitral severa y válvula mitral protésica. Se realizó un análisis exploratorio inicial de tipo descriptivo mediante frecuencias y porcentajes para variables categóricas y medidas de tendencia central (media o mediana según la distribución normal de los datos) para variables cuantitativas. Posteriormente, se compararon los diferentes grados de disfunción diastólica respecto a la edad mediante prueba t-Student y respecto a variables categóricas mediante $\chi^{2}$. Resultados: Se reclutaron un total de 610 pacientes, de los cuales 75 fueron retirados de la muestra por cumplir criterios de exclusión, se incluyeron 535 pacientes de los cuales $198(37 \%)$ se estratificaron como disfunción diastólica grado I, 63 $(11,7 \%)$ grado II, $29(5,4 \%)$ grado III, 38 (7,1\%) indeterminado y $207(38,6 \%)$ sin disfunción diastólica. La edad de los pacientes estratificados en cualquier grado de disfunción diastólica fue significativamente mayor en comparación con los pacientes sin disfunción diastólica $(p<0,001)$. La hipertensión arterial fue la comorbilidad asociada más importante en los pacientes con disfunción diastólica grado I (en 54\%), grado II (en 50\%), mientras que la enfermedad coronaria fue

\section{Palabras clave:}

Disfunción diastólica, patrones de llenado, ecocardiografía. 
la más frecuente comorbilidad encontrada en la presencia de disfunción diastólica grado III (en 48\%). La relación E/e' mayor a 14 estuvo presente en el 35\% de los casos con disfunción diastólica grado II y en el $72 \%$ de los casos con disfunción diastólica grado III. Conclusión: La disfunción diastólica es un hallazgo muy frecuente $(323 / 530,61 \%)$. La hipertensión arterial fue la comorbilidad más frecuentemente asociada en los grados I, II de disfunción diastólica, mientras que la enfermedad coronaria con el grado III. El aumento de la relación E/e' >14 como parámetro indicativo de aumento de la presión de llenado estuvo presente en el 35\% de los pacientes con disfunción diastólica grado II y en el $72 \%$ de los pacientes con disfunción grado III. La función diastólica se clasificó como indeterminada en el 7,1\% de la población total de pacientes. Las Guías ASE/EACVI permitieron la clasificación en diferentes grados de disfunción diastólica en la mayoría de los pacientes evaluados (94\%). Otros métodos de evaluación diagnóstica serán necesarios para clasificar adecuadamente al grupo de pacientes con función diastólica indeterminada.

\section{Introducción}

a insuficiencia cardíaca es un importante problema de salud pública que representa más de 1 millón de hospitalizaciones anuales en los Estados Unidos, y múltiples visitas a salas de emergencias[1]. En los pacientes que debutan con disnea, el diagnóstico clínico puede ser desafiante porque en la insuficiencia cardíaca los síntomas son relativamente inespecíficos y pueden deberse a causas no cardíacas. En ese sentido, las imágenes cardiacas pueden ser útiles para alcanzar el diagnóstico correcto y posteriormente guiar el manejo terapéutico del paciente[1]. El ecocardiograma suele ser la primera prueba de imagen que se obtiene, ya que proporciona información inmediata sobre el tamaño y función del ventrículo izquierdo, incluyendo la fracción de eyección del ventrículo izquierdo (FEVI) y la función valvular[2],[3]. Sin embargo, la presencia de una FEVI normal no excluye una etiología cardíaca para la disnea, ya que aproximadamente el $50 \%$ de los pacientes con falla cardiaca solo tienen una fracción de eyección levemente reducida o generalmente está preservada quedando categorizados con el término insuficiencia cardíaca con fracción de eyección preservada o disfunción diastólica sintomática[4].

La disfunción diastólica ventricular izquierda con fracción de eyección preservada es una patología frecuente en la población mundial con importantes implicaciones en cuanto a mortalidad y morbilidad[3],[4]. Las Guías ASE/EACVI 2016, recientemente publicadas para evaluar función diastólica, tratan de simplificar en una forma más comprensiva la evaluación ecocardiográfica de esta patología compleja[5]. No existen hasta ahora estudios en Colombia que evalúen la factibilidad y reproducibilidad de las Guías para evaluar la disfunción diastólica. No conocemos las características sociodemográficas y clínicas de nuestra población adulta con disfunción diastólica, evaluados con ecocardiografía transtorácica ni tampoco el patrón de aumento de presiones de llenado más frecuentemente encontrado, así como la utilidad de estas Guías para poder estratificar a los pacientes en cualquier tipo de disfunción.

La finalidad del presente estudio es describir y analizar la utilidad de las últimas Guías de función diastólica publicadas ASE/EACVI 2016[5] para estratificar de una manera sencilla y descriptiva los diferentes patrones de llenado y grados de disfunción diastólica del ventrículo izquierdo en un centro de tercer nivel en la ciudad de Medellín, Colombia.

\section{Metodología}

\section{Tipo de estudio}

Se realizó un estudio prospectivo observacional entre febrero y junio de 2017.

\section{Población del estudio}

Se decidió realizar el estudio incluyendo pacientes que consultaron al laboratorio de ecocardiografía de la clínica Medellín ubicado en la ciudad de Medellín, Colombia, en el cual se realizaron estudios ecocardiográficos transtorácicos.

A continuación, describimos los principales criterios de exclusión de nuestro estudio, que correspondieron al $13 \%$ de la muestra total de pacientes ( 75 de 610 pacientes) 


\section{Procedimientos}

Los autores analizaron los ecocardiogramas 2D realizados diariamente en el laboratorio de ecocardiografía de la clínica Medellín y extrajeron cada una de las mediciones necesarias para la estratificación según las Guías ASE/EACVI 2016[5]. El ecocardiograma fue realizado mediante la vista apical de 4 cámaras para obtener las velocidades de flujo mitral y del flujo venoso pulmonar según las recomendaciones de las Guías[5],[6]. En cuanto a los parámetros hemodinámicos, las primeras mediciones incluyeron las velocidades sistólicas (S), diastólica temprana y diastólica tardía a nivel del anillo mitral. La velocidad anular diastólica temprana ha sido expresada como Ea, Em, $\mathrm{E}^{\prime}$ o e', y la velocidad diastólica tardía como Aa, Am, $A^{\prime}$ o $a^{\prime}$. Los autores del presente documento prefieren el uso de e' y a'. Una vez adquiridas las velocidades de flujo mitral, las velocidades anulares y los intervalos de tiempo, es posible computar intervalos y cocientes adicionales. Estos cocientes incluyeron la relación e'/a' anular y E/e' (velocidad de flujo mitral E sobre e' de Doppler tisular)[7].

\section{Estratificación}

Primero se procedió a definir el diagnóstico de disfunción diastólica, posteriormente se realizó la estratificación de acuerdo a las guías ASE/EACVI 2016[5] utilizando el algoritmo de la Figura 1, en el cual se tienen 4 criterios de evaluación de la función diastólica en pacientes con FEVI normal:1. E/e'> 14, 2. Velocidad e'septal $<7 \mathrm{~cm} / \mathrm{s}$ o velocidad e'lateral $<10 \mathrm{~cm} / \mathrm{s}, 3$. Velocidad regurgitación tricuspidea (RT) $>2,8 \mathrm{~m} / \mathrm{s}$ y 4 . Volumen indexado de la aurícula izquierda $>34 \mathrm{~mL} / \mathrm{m}^{2}$.

Si de estos criterios $<50 \%$ son positivos, la función diastólica se considera normal, si son positivos > $50 \%$, hay disfunción diastólica presente y si solo el $50 \%$ de los criterios son positivos, se considera función diastólica indeterminada. El esquema de estratificación (Figura 2) consiste en: leve o grado I (patrón de relajación prolongada), moderada o grado II y severa (Ilenado restrictivo) o grado III. Fueron definidas de acuerdo a los siguientes criterios:

Grado I: Si el cociente E/A mitral es $<0,8$ más onda $\mathrm{E}<50 \mathrm{~cm} / \mathrm{s}$, se considera como disfunción diastólica grado I o presión de Al normal. Cuando el cociente E/A mitral $<0,8$ más onda $E>50 \mathrm{~cm} / \mathrm{s}$ o cociente E/A $>0,8-<2,3$ criterios deben evaluarse (E/e'> 14, velocidad $\mathrm{RT}>2,8 \mathrm{~m} / \mathrm{s}$, volumen indexado de $\mathrm{Al}>34 \mathrm{~mL}$ $\mathrm{m}^{2}$ ). Si 2 ó 3 criterios de 3 posibles son negativos, se considera también como disfunción diastólica grado I.

Grado II: Cociente E/A mitral $<0,8$ más onda $E>$ $50 \mathrm{~cm} / \mathrm{s}$ o cociente $\mathrm{E} / \mathrm{A}>0,8-<2,3$ criterios deben evaluarse (E/e'> 14, velocidad RT $>2,8 \mathrm{~m} / \mathrm{s}$, volumen indexado de $\mathrm{Al}>34 \mathrm{~mL} / \mathrm{m}^{2}$. Si 2 ó 3 de 3 posibles son positivos, hay aumento de la presión en la aurícula izquierda y se cataloga como disfunción diastólica

\section{Algoritmo de Evaluación en Pacientes con FEVI Normal}

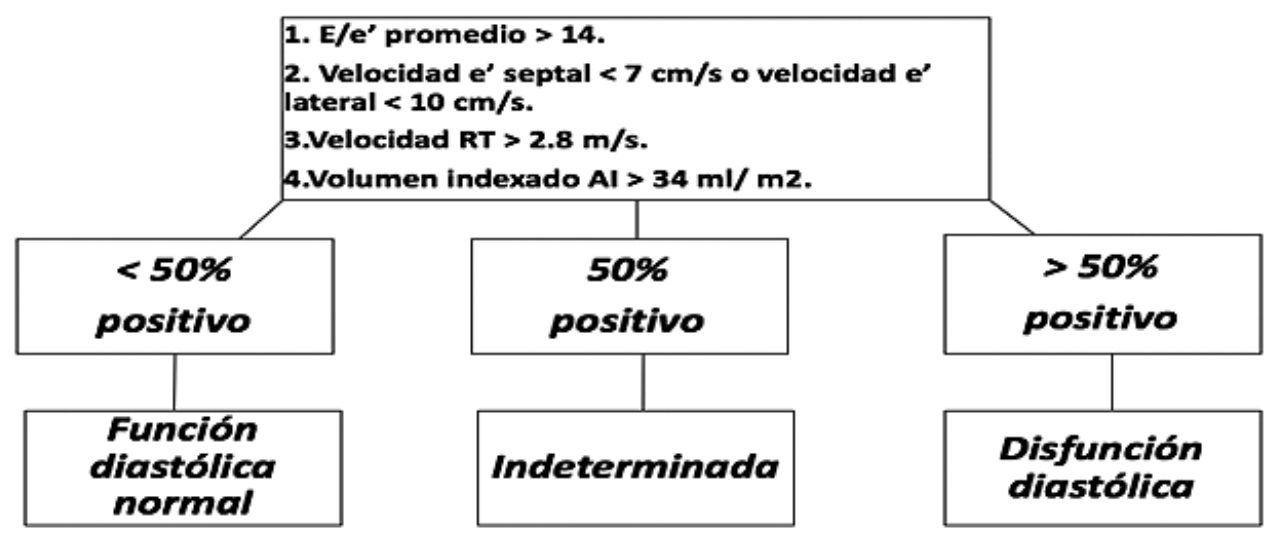

Figura 1. Algoritmo para el diagnóstico de la disfunción diastólica del VI en sujetos con FEVI normal. Tomado de Nagueh SF, et al. Recommendations for the evaluation of left ventricular diastolic function by echocardiography. Journal of the American Society of Echocardiography 2016;29:277-314 


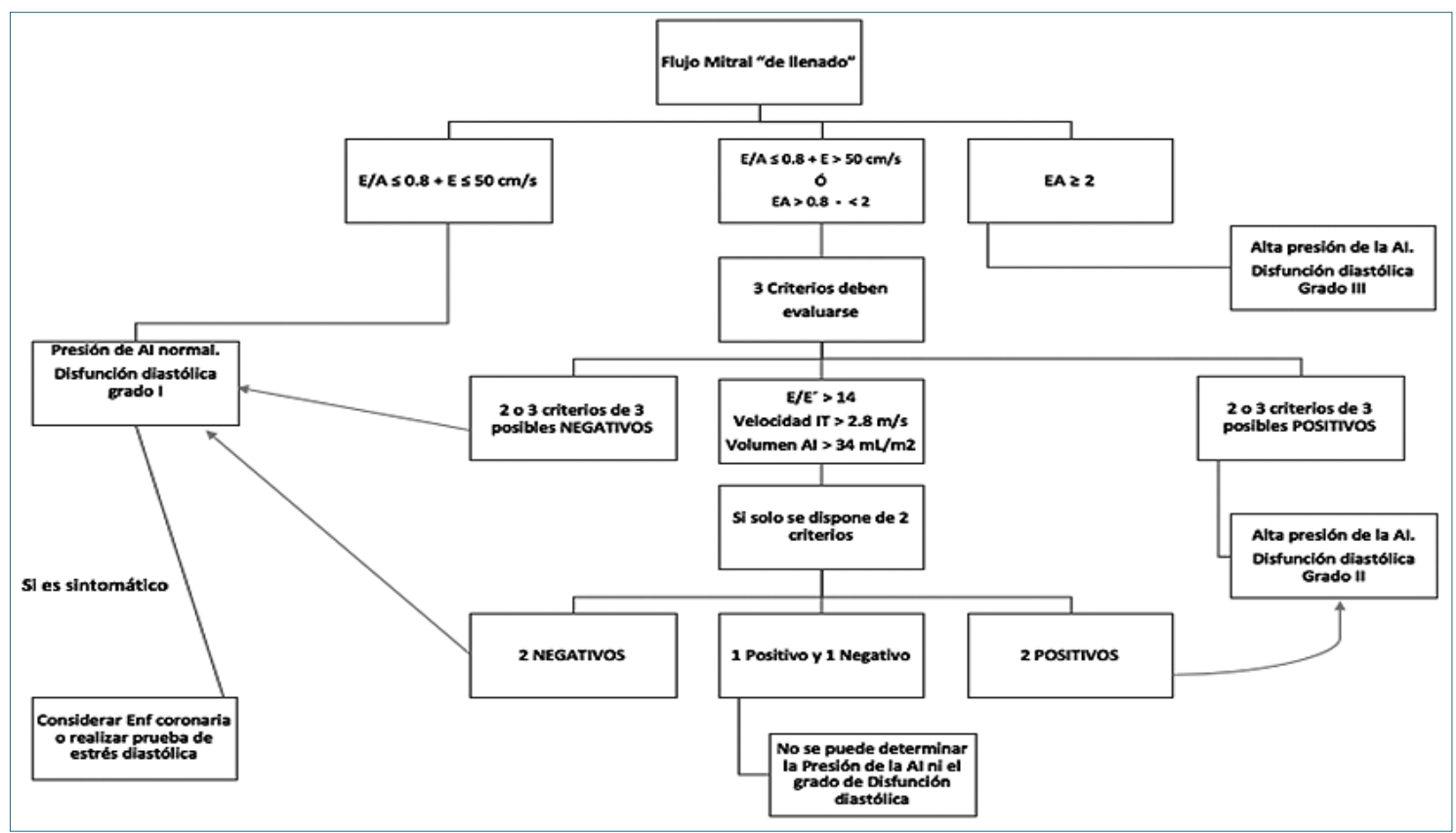

Figura 2. Algoritmo para la estimación de las presiones de llenado de la Al y graduación de la función diastólica del VI en pacientes con FEVI deprimida y en pacientes con enfermedad miocárdica y FEVI normal. (*: Presión en la aurícula izquierda es indeterminado si solo está disponible 1 de 3 parámetros. Tomado de Nagueh SF, et al. Recommendations for the evaluation of left ventricular diastolic function by echocardiography. Journal of the American Society of Echocardiography 2016;29:277314.

grado II. Si solo se dispone de 2 criterios, y los 2 son negativos, se considera como disfunción diastólica grado I o presión de Al normal. Si solo se dispone de 2 criterios y los 2 son positivos, también se considera disfunción diastólica grado II o presión elevada de Al.

Grado III: Cociente E/A $\geq 2$. Se considera que hay aumento de presión en la aurícula izquierda y se catalogó como disfunción diastólica grado III.

Indeterminado: Cuando la fracción de eyección está normal, se tienen en cuenta 4 variables (promedio E/ $\mathrm{e}^{\prime}>14$, velocidad de regurgitación tricuspidea $>2,8$ $\mathrm{m} / \mathrm{s}$, volumen de aurícula izquierda indexada $>34 \mathrm{~mL}$ $\mathrm{m}^{2}$, velocidad e'septal $<7 \mathrm{~cm} / \mathrm{s}$ o velocidad e' late$\mathrm{ral}<10 \mathrm{~cm} / \mathrm{s})$. Si 2 de 4 son positivos se catalogó como indeterminada. Si la fracción de eyección está disminuida, o hay enfermedad miocárdica se tienen en cuenta 3 variables (promedio E/e' $>14$, velocidad de regurgitación tricuspidea $>2,8 \mathrm{~m} / \mathrm{s}$, volumen de aurícula izquierda indexada $>34 \mathrm{~mL} / \mathrm{m}^{2}$ ).

Si de las 3 variables, solo se tienen 2 criterios disponibles, y 1 es positivo y el otro es negativo queda tipificado como disfunción diastólica indeterminada.
Relación de S / D de la vena pulmonar $<1$ aplicable para concluir que la presión en la aurícula izquierda esta elevada en pacientes con FEVI deprimida).

\section{Análisis estadístico}

Se realizó un análisis exploratorio inicial de tipo descriptivo mediante frecuencias y porcentajes para variables categóricas y medidas de tendencia central (media o mediana según la distribución normal de los datos) para variables cuantitativas. Posteriormente, se compararon los diferentes grados de disfunción diastólica respecto a la edad mediante prueba t-Student y respecto a otras variables categóricas (i.e., comorbilidades como hipertensión arterial, diabetes, antecedente de infarto agudo de miocardio, hipotiroidismo, entre otras) mediante chi-cuadrado.

\section{Resultados}

Se reclutaron un total de 610 pacientes, de los cuales 75 fueron retirados de la muestra por cumplir 
criterios de exclusión, se incluyeron 535 pacientes. En la Tabla 1 se pueden observar las características sociodemográficas de los pacientes incluidos en nuestro estudio.

Del total de pacientes, en el $60 \%$ se encontró algún grado de disfunción diastólica discriminado así: 198 (37\%) disfunción diastólica grado I, 63 (11,7\%) grado II, $29(5,4 \%)$ grado III, $38(7,1 \%)$ indeterminado y 207 (38,6\%) sin disfunción diastólica. De los pacientes indeterminados, 5 (13,15\%) tenía fracción de eyección moderadamente preservada (FEVI 40\%$50 \%), 33(86,8 \%)$ tenían falla cardiaca con fracción de eyección preservada ( $\mathrm{FEVI}>50 \%$ ), ningún paciente con $\mathrm{FEVI}<40 \%$ quedó como indeterminado. La edad de los pacientes estratificados en cualquier grado de disfunción diastólica fue significativamente mayor en comparación con los pacientes sin disfunción diastólica $(p<0,001)$. En la Figura 2 se logra observar la distribución etaria de los pacientes de acuerdo a cada grado de disfunción diastólica.

La hipertensión arterial fue la comorbilidad asociada más importante en los pacientes con disfunción diastólica grado I (en 54\%), grado II (en 50\%), mientras que la enfermedad coronaria fue la más frecuente comorbilidad encontrada en la presencia de disfunción diastólica grado III (en 48\%). En la Figura 3 se ha graficado la distribución de las principales comorbilidades por grado de disfunción diastólica y en la Tabla 2 la descripción de la muestra.

\begin{tabular}{lc}
\hline \multicolumn{2}{c}{ Tabla 1. Criterios de exclusión } \\
\hline Criterio & Frecuencia, (\%) \\
Insuficiencia mitral severa & $25(33,3 \%)$ \\
Fibrilación auricular & $24(32 \%)$ \\
Marcapasos & $15(20 \%)$ \\
Prótesis mitral & $11(14,6 \%)$ \\
Total & 75 \\
\hline
\end{tabular}

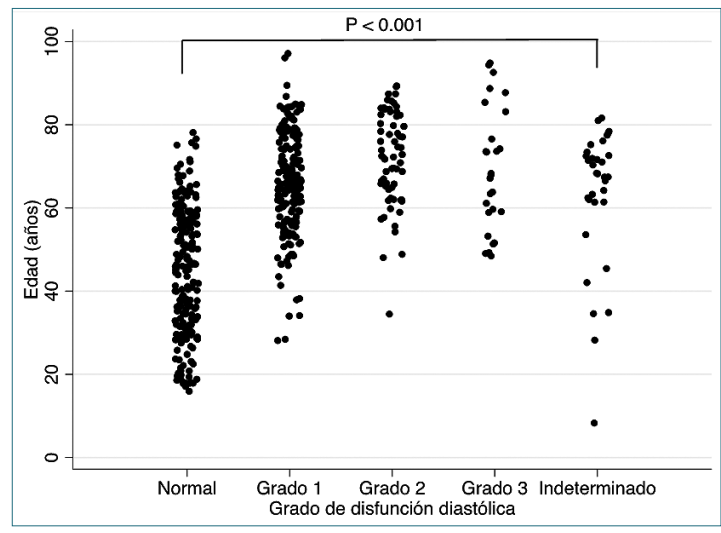

Figura 2. Distribución de edad según grado de disfunción diastólica.

\begin{tabular}{|c|c|c|c|c|c|c|c|}
\hline & \multirow[b]{2}{*}{$\begin{array}{l}\text { Total } \\
(n=530)\end{array}$} & \multicolumn{6}{|c|}{ Disfunción diastólica } \\
\hline & & $\begin{array}{l}\text { Grado } 1 \\
(n=198)\end{array}$ & $\begin{array}{l}\text { Grado II } \\
(n=63)\end{array}$ & $\begin{array}{l}\text { Grado III } \\
(n=29)\end{array}$ & $\begin{array}{l}\text { Indetermina- } \\
\text { do }(n=38)\end{array}$ & $\begin{array}{l}\text { Normal } \\
(n=207)\end{array}$ & $P$ \\
\hline Edad, años & $58,5 \pm 17,5$ & $65,7 \pm 12,5^{\star}$ & $72,3 \pm 10,3^{*}$ & $69,1 \pm 14,1$ * & $63,1 \pm 16,4^{*}$ & $45,4 \pm 15,2$ & $<0,001$ \\
\hline Género & & & & & & & 0,233 \\
\hline Masculino & $326(61,5 \%)$ & $77(24 \%)$ & $28(9 \%)$ & $14(4 \%)$ & $20(5 \%)$ & $70(21 \%)$ & \\
\hline Femenino & $204(3,85 \%)$ & $121(59 \%)$ & $35(17 \%)$ & $15(7,3 \%)$ & $18(8 \%)$ & $137(67 \%)$ & \\
\hline Peso, kg & $70,7 \pm 14,7$ & $72,9 \pm 15,2$ & $67,0 \pm 14,3$ & $65,7 \pm 15,1$ & $73,7 \pm 14,1$ & $70,3 \pm 13,9$ & 0,014 \\
\hline Talla, cm & $166,9 \pm 83,1$ & $174 \pm 134$ & $161 \pm 10,1$ & $162 \pm 7,6$ & $164 \pm 8,8$ & $162 \pm 8,8$ & 0,640 \\
\hline \multicolumn{8}{|l|}{ Comorbilidades } \\
\hline $\begin{array}{l}\text { Enfermedad co- } \\
\text { ronaría }\end{array}$ & $88(16,5 \%)$ & $39(20,7 \%)^{\star}$ & $17(26,5 \%)^{\star}$ & $15(48,1 \%)^{\star}$ & $7(21,2 \%)^{\star}$ & $10(4,1 \%)$ & $<0,001$ \\
\hline UTA & $228(44,5 \%)$ & $104(54 \%)^{\star}$ & $32(50 \%)^{*}$ & $3(11 \%)$ & $18(47,2)$ & $76(38 \%)$ & 0,223 \\
\hline EPOC & $31(5,7 \%)$ & $14(6,3 \%)$ & $5(7,83 \%)$ & $2(7,4 \%)$ & $0(0 \%)$ & $10(5,1 \%)$ & 0,122 \\
\hline DM & $19(3,5 \%)$ & $7(3,7 \%)$ & $5(7,8 \%)$ & $4(14,8 \%)$ & $1(3,03 \%)$ & $2(1 \%)$ & 0,431 \\
\hline Otros" & $152(29 \%)$ & $28(14,8 \%)$ & $4(7 \%)$ & $5(18,5 \%)$ & $12(36,3 \%)$ & $103(50 \%)$ & \\
\hline
\end{tabular}

*Valores de $p<0,05$ comparando pacientes sin disfunción diastólica versus cualquier grado de disfunción.

**Otros: ACV, TEP, Cáncer. CIA. CIV, Hipotiroidismo. Dolor torácico, preqx. lupus. 


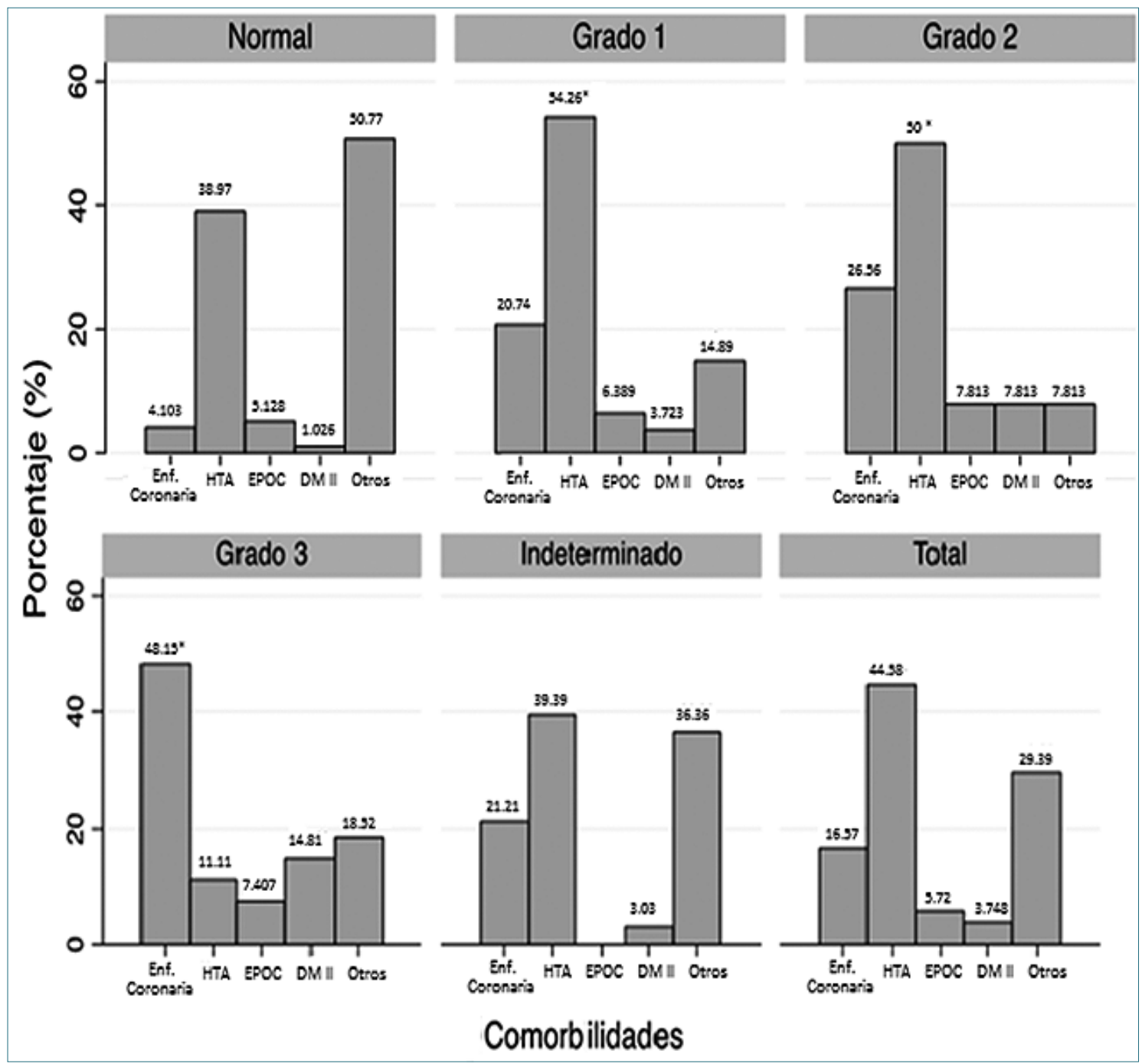

Figura 3. Principales comorbilidades por grado de disfunción diastólica.

La relación E/e' mayor a 14 estuvo presente en el $35 \%$ de los casos con disfunción diastólica grado $\|$ y en el $72 \%$ de los casos con disfunción diastólica grado III.

\section{Discusión}

En nuestra cohorte las Guías ASE/EACVI 2016 permitieron la clasificación de la función diastólica en diferentes grados en la mayoría de los pacientes evaluados (94\%). El aumento de la relación E/e' > 14 como parámetro indicativo del aumento de la presión de llenado del VI estuvo presente en el $45 \%$ de los pacientes con disfunción diastólica grado II y en el $72 \%$ de los pacientes con disfunción grado III como se observó en el estudio de Balaney[8] Khouri 2004[9], Andersen et al[10] y Flachskampf[11], La relación E/e '> 14 es un predictor útil para la evaluación de las presiones de llenado del ventrículo izquierdo en la práctica clínica, sin embargo, su exactitud ha sido cuestionada en los pacientes descompensados con insuficiencia cardiaca sistólica avanzada en los que esta relación puede no ser tan confiable para predecir las presiones de llenado intracardíacas, particularmente en aquellos con mayores volúmenes del VI, índices cardiacos más 


\begin{tabular}{|c|c|c|c|c|c|}
\hline & $\begin{array}{c}<30 \\
(n=41)\end{array}$ & $\begin{array}{c}30-49 \\
(n=103)\end{array}$ & $\begin{array}{c}50-69 \\
(n=229)\end{array}$ & $\begin{array}{c}70-89 \\
(n=144)\end{array}$ & $\begin{array}{c}>90 \\
(n=6)\end{array}$ \\
\hline$E / A \leq 0,8, E<50 \mathrm{~cm} / \mathrm{seg}$ & 0 & 3 & 41 & 19 & 3 \\
\hline$E / A \leq 0,8, E>50 \mathrm{~cm} / \mathrm{seg}$ & 4 & 11 & 83 & 70 & 0 \\
\hline E/A $0,8-2$ & 35 & 82 & 99 & 49 & 2 \\
\hline$E / A>2$ & 1 & 3 & 11 & 6 & 1 \\
\hline$E / e^{\prime}>14$ & 0 & 0 & 15 & 27 & 3 \\
\hline RT vel $>2,8 \mathrm{~m} / \mathrm{s}$ & 2 & 11 & 75 & 74 & 6 \\
\hline RT desconocida & 6 & 16 & 51 & 19 & 0 \\
\hline Al indexada $>34 \mathrm{~mL} / \mathrm{m}^{2}$ & 4 & 14 & 82 & 65 & 3 \\
\hline Onda e'lat $<10 \mathrm{~cm} / \mathrm{s}$ & 3 & 7 & 98 & 103 & 3 \\
\hline Onda e'septal $<7 \mathrm{~cm} / \mathrm{s}$ & 0 & 6 & 84 & 87 & 4 \\
\hline TD $160-200 \mathrm{~ms}$ & 2 & 11 & 55 & 58 & 5 \\
\hline
\end{tabular}

E: La velocidad de la onda E refleja el gradiente de presión AI-VI durante la diástole temprana y se ve afectada por alteraciones en la tasa de relajación del VI y la presión de la aurícula izquierda.

A: La velocidad de la onda A refleja el gradiente de presión de AI-VI durante la diástole tardía, que se ve afectada por la distensibilidad del ventrículo izquierdo y función contráctil de la aurícula izquierda.

Relación E/A: La relación E/A de flujo de entrada mitral se utiliza para identificar los patrones de llenado del ventrículo izquierdo: relajación normal y alterada, pseudonormal y llenado restrictivo.

TD: tiempo desaceleración: influenciado por la relajación del VI, las presiones diastólicas y la rigidez del VI.

e': velocidad diastólica temprana obtenida por Doppler de tejidos. Una asociación significativa está presente entre e' y la constante de tiempo de relajación del VI (tau). Los determinantes hemodinámicos de la velocidad e 'incluyen relajación del $\mathrm{VI}$, fuerzas de restauración y presión de llenado VI.

Relación E/e': La relación E / e' puede ser utilizada para predecir las presiones de llenado del VI.

Regurgitación tricuspidea RT: Obtenido con Doppler de onda continua (CW) de la velocidad del jet de regurgitación tricuspidea. En ausencia de enfermedad pulmonar, el aumento de la presión sistólica de la arteria pulmonar sugiere incremento de la presión en la aurícula izquierda.

deteriorados y la presencia de resincronización cardíaca[12]. El estudio de Andersen y col documentó que, pese a encontrar una aurícula izquierda aumentada de tamaño y una velocidad de regurgitación tricuspidea mayor a 2,8, $\mathrm{m} / \mathrm{s}$, las presiones de llenado del ventrículo izquierdo se encontraron elevadas solo en 11 de 19 pacientes quedando 9 tipificados como disfunción diastólica II, pero erróneamente, ya que en el cateterismo cardiaco se encontró presiones de llenado normales, aclarando que fueron 19 pacientes de una corte de 450 (4,2\%)[10].

En nuestra cohorte, una relación E/e' $>14$ estuvo presente en el $72 \%$ de los pacientes con disfunción diastólica tipo III, sin embargo, en nuestro estudio, no se realizó cateterismo cardiaco para demostrar un incremento de la presión de llenado. Las guías de función diastólica 2016 tuvieron en cuenta los puntos de corte de normalidad según los resultados del estudio NORRE en 449 sujetos sin evidencia de patología cardiovascular[13], sin embargo, como Dencker y col.[14] sugieren, aún puede ser prematuro definir valores de corte normales, dado que hay variabilidad entre los diferentes estudios y aún no es muy claro los límites para definir anormalidad como referencia estándar.

A diferencia de lo sugerido en las Guías 2016, Mitter y col[15], toman en cuenta los cambios de la velocidad e'lateral según la edad (edad > 55 años, de 55 a 65 y > 65 años) y consideran el volumen de la aurícula izquierda anormal mayor de $28 \mathrm{~mL} / \mathrm{m}^{2}$, asi como puntos de corte a la relación E/e' ( < a 8 normal, 8 a 12 indeterminada y mayor a 12 anormal) para definir incremento de la presión de llenado del VI.

En nuestro estudio encontramos que la función diastólica quedó tipificada como indeterminada en el $7,1 \%$ de la población, tres puntos porcentuales menos que lo encontrado en el estudio Balaney y col[8], y al igual que este el mayor número de pacientes en el grupo indeterminado fue con fracción de eyección preservada. 
La disfunción diastólica ha sido clasificada de múltiples formas y bajo muchos criterios, en las Guías ASE/ EACVI 2009[3], se emiten conceptos fisiológicos, se habla de nuevas metodologías, se dan valores numéricos de corte, mejor abordaje a problemas complejos, y se informan diagramas abiertos con múltiples mediciones posibles, sin embargo, había mayor dificultad para una adecuada clasificación por el gran número de variables analizadas. Las Guías ASE/EACVI 2016[5] son publicadas 7 años después y la piedra angular de estas nuevas Guías es la simplificación del abordaje con una mayor integración a los datos clínicos[6],[7].

Consideramos que el uso de las Guías ASE/EACVI 2016 está justificado en la práctica clínica rutinaria para realizar diagnóstico y estratificación de los pacientes con disfunción diastólica, lo cual tiene implicaciones pronósticas, a mayor compromiso de la función diastólica, peor es el pronóstico. Consideramos que en todo paciente al cual se realiza un estudio ecocardiográfico debe mencionarse en el informe clínico final la evaluación de la función diastólica.

\section{Limitaciones}

El presente estudio tiene varias limitaciones que deben ser mencionadas, la más importante, no hubo una comparación con presiones de llenado y función diastólica evaluada por métodos invasivos para corroborar el grado de disfunción diastólica determinado por ecocardiografía. Sin embargo, el estudio de Andersen y col[10] claramente demostró que la evaluación de la función diastólica según las Guías ASE/ EACVI 2016 es correcta en la mayoría de los pacientes estudiados invasivamente.

La estratificación de la función diastólica sugerida por las guías presenta imperfecciones ya que existen falsos positivos que son tomados como casos de disfunción diastólica cuando en realidad no lo son como lo sugiere el estudio NORRE[13], por ejemplo, 1) Pacientes con volumen indexado de aurícula izquierda $>34 \mathrm{~mL} / \mathrm{m}^{2}$ estuvo presente en el 10,5\% (31 de 294 sujetos normales) sin aumento de las presiones de llenado; 2) Presión sistólica de arteria pulmonar $>35$ $\mathrm{mmHg}$ en el 0,3\% (1 de 294 sujetos) con presiones de llenado normales; 3) El promedio E/e' > 14 se presentó en el $1 \%$ (4 sujetos de 420) sin alteraciones en las presiones de llenado; 4) La velocidad e' del anillo mitral tiene limitaciones para definir el grado de disfunción diastólica, la onda e'septal $<8 \mathrm{~cm} / \mathrm{s}$ se presentó en el 19,3\% (86 de 446 sujetos) sin ninguna alteración del patrón de llenado, así como la onda é lateral menor a $10 \mathrm{~cm} / \mathrm{s}$ se encontró en el 16,9\% (74 de 438 sujetos normales), sin que esto se correlacionara con trastornos del Ilenado[10]-[11]. En nuestro estudio, tampoco se realizó ecocardiograma de estrés con ejercicio a todos los pacientes sintomáticos y con presión de aurícula izquierda normal, para definir la presencia de disfunción diastólica[5]. Adicionalmente y de considerable importancia, el análisis correcto de la función diastólica siempre tiene que ir de la mano con el contexto clínico del paciente para abordar cualquier decisión diagnóstica y terapéutica. Como lo expresan explícitamente las guías 2016, hay situaciones clínicas especiales donde no son aplicables los algoritmos propuestos, por ejemplo, pacientes con miocardiopatía hipertrófica, calcificación severa del anillo mitral, estenosis/insuficiencia mitral, insuficiencia aórtica, fibrilación/flutter atrial, hipertensión pulmonar primaria, estimulación con marcapasos, entre otras[5].

\section{Conclusiones}

La estratificación de la función diastólica según las nuevas Guías ASE/EACVI 2016, puede realizarse de forma rutinaria en el laboratorio de ecocardiografía. En la mayoría de los pacientes, pudo clasificarse en diferentes grados de severidad. Sin embargo, en un grupo considerable de pacientes $(7,1 \%)$, la función diastólica fue indeterminada, por lo cual, serán necesarios métodos diagnósticos adicionales para una correcta evaluación.

El uso de las Guías ASE/EACVI 2016 está justificado en la práctica clínica rutinaria para realizar diagnóstico y estratificación de los pacientes con disfunción diastólica. Consideramos que en todo paciente sometido a un estudio ecocardiográfico debe mencionarse en el informe clínico final la evaluación de la función diastólica.

\section{Referencias}

1. Kane GC, Karon BL, Mahoney DW, Redfield MM, Roger VL,
Burnett JC Jr, et al. Progression of left ventricular diastolic dysfunction and risk of heart failure. JAMA. 2011 Aug;306(8):856-63.
2. Patel KD, Collier P, Klein AL. Modern Assessment of Diastolic Function. Curr Cardiovasc Imaging Rep. 2016;9(10):28. 
3. Nagueh SF, Appleton CP, Gillebert TC, Marino PN, Oh JK, Smiseth OA, et al. Recommendations for the evaluation of left ventricular diastolic function by echocardiography. J Am Soc Echocardiogr. 2009 Feb;22(2):107-33.

4. AlJaroudi WA, Thomas JD, Rodriguez LL, Jaber WA. Prognostic value of diastolic dysfunction: state of the art review. Cardiol Rev. 2014 Mar-Apr;22(2):79-90.

5. Nagueh SF, Smiseth OA, Appleton CP, Byrd BF 3rd, Dokainish $H$, Edvardsen $T$, et al. Recommendations for the evaluation of left ventricular diastolic function by echocardiography: an update from the American Society of Echocardiography and the European Association of Cardiovascular Imaging. J Am Soc Echocardiogr. 2016 Apr;29(4):277-314.

6. Kuwaki H, Takeuchi M, ChienChia Wu V, Otani K, Nagata $Y$, Hayashi A, et al. Redefining diastolic dysfunction grading: combination of E/A $\leq 0.75$ and deceleration time $>140 \mathrm{~ms}$ and $\mathrm{E} / \varepsilon^{\prime} \geq 10$. JACC Cardiovasc Imaging. 2014 Aug;7(8):749-58.

7. Sharifov OF, Schiros CG, Aban I,
Denney TS, Gupta H. Diagnostic accuracy of tissue Doppler index E/e'for evaluating left ventricular filling pressure and diastolic dysfunction/heart failure with preserved ejection fraction: a systematic review and metaanalysis. J Am Heart Assoc. 2016 Jan;5(1):e002530.

8. Bhavna Balaney MD. Diego Medvedofsky, Invasive Validation of the Echocardiographic Assessment of Left Ventricular Filling Pressures Using the 2016 Diastolic Guidelines: Head-toHead Comparison with the 2009 Guidelines. J Am Soc Echocardiogr. 2018;1(1):79-88.

9. Khouri SJ, Maly GT, Suh DD, Walsh TE. A practical approach to the echocardiographic evaluation of diastolic function. J Am Soc Echocardiogr. 2004 Mar;17(3):290-7.

10. Andersen OS, Smiseth $O A$, Dokainish $\mathrm{H}$, Abudiab MM, Schutt RC, Kumar A, et al. Estimating Left Ventricular Filling Pressure by Echocardiography. J Am Coll Cardiol. 2017 Apr;69(15):1937-48.

11. Flachskampf FA, Baron $T$. Echocardiographic algorithms for detecting elevated diastolic pressures. J Am Coll Cardiol. 2017 Apr;69(15):1949-51.

12. Mullens W, Borowski AG, Curtin RJ, Thomas JD, Tang WH. Tissue Doppler imaging in the estimation of intracardiac filling pressure in decompensated patients with advanced systolic heart failure. Circulation. 2009 Jan;119(1):62-70.

13. Kou S, Caballero L, Dulgheru R, Voilliot D, De Sousa C, Kacharava $G$, et al. Echocardiographic reference ranges for normal cardiac chamber size: results from the NORRE study. Eur Heart J Cardiovasc Imaging. 2014 Jun;15(6):680-90.

14. Magnus Dencker and Martin Stagmo. Reported normal values and weighted means for commonly used echocardiography pulsed Doppler and tissue Doppler measurements. Clin Physiol Funct Imaging Scandinavian Society of Clinical Physiology and Nuclear Medicine; 2017.

15. Mitter SS, Shah SJ, Thomas JD. A Test in Context. Journal of the American College of Cardiology [Internet]. Elsevier BV; 2017 Mar;69(11):1451-64. 